(2) Open Access Full Text Article

ORIGINAL RESEARCH

\title{
Impact of COPD diagnosis timing on clinical and economic outcomes: the ARCTIC observational cohort study
}

This article was published in the following Dove Press journal:

International Journal of Chronic Obstructive Pulmonary Disease

\author{
Kjell Larsson' \\ Christer Janson ${ }^{2}$ \\ Björn Ställberg ${ }^{3}$ \\ Karin Lisspers ${ }^{3}$ \\ Petter Olsson ${ }^{4}$ \\ Konstantinos Kostikas ${ }^{5}$ \\ Jean-Bernard Gruenberger ${ }^{5}$ \\ Florian S Gutzwiller ${ }^{5}$ \\ Milica Uhde ${ }^{6}$ \\ Leif Jorgensen ${ }^{7}$ \\ Gunnar Johansson ${ }^{3}$ \\ 'Work Environment Toxicology, \\ Karolinska Institutet, Stockholm, Sweden; \\ ${ }^{2}$ Department of Medical Sciences: \\ Respiratory, Allergy and Sleep Research, \\ Uppsala University, Uppsala, Sweden; \\ ${ }^{3}$ Public Health and Caring Sciences, \\ Family Medicine and Preventive Medicine, \\ Uppsala University, Uppsala, Sweden; \\ ${ }^{4}$ Novartis $A B$, Täby, Sweden; ${ }^{5}$ Novartis \\ Pharma AG, Basel, Switzerland; ' $I Q V I A$, \\ Solna, Sweden; ${ }^{7} \mathrm{IQVIA}$, Copenhagen, \\ Denmark
}

Correspondence: Kjell Larsson

Work Environment Toxicology,

Karolinska Institutet, Stockholm SE-17I

77, Sweden

Tel +46705820763

Email kjell.larsson@ki.se
Purpose: Assess the clinical and economic consequences associated with an early versus late diagnosis in patients with COPD.

Patients and methods: In a retrospective, observational cohort study, electronic medical record data (2000-2014) were collected from Swedish primary care patients with COPD. COPD indicators (pneumonia, other respiratory diseases, oral corticosteroids, antibiotics for respiratory infections, prescribed drugs for respiratory symptoms, lung function measurement) registered prior to diagnosis were applied to categorize patients into those receiving early ( 2 or less indicators) or late diagnosis ( 3 or more indicators registered $>90$ days preceding a COPD diagnosis). Outcome measures included annual rate of and time to first exacerbation, mortality risk, prevalence of comorbidities and health care utilization.

Results: More patients with late diagnosis $(n=8827)$ than with early diagnosis $(n=3870)$ had a recent comorbid diagnosis of asthma $(22.0 \%$ vs $3.9 \%$; $P<0.0001)$. Compared with early diagnosis, patients with late diagnosis had a higher exacerbation rate (hazard ratio [HR] 1.89, 95\% confidence interval $[\mathrm{CI}]: 1.83-1.96 ; P<0.0001)$ and shorter time to first exacerbation (HR $1.61,95 \% \mathrm{CI}$ : $1.54-1.69 ; P<0.0001)$. Mortality was not different between groups overall but higher for late versus early diagnosis, after excluding patients with past asthma diagnosis (HR 1.10, 95\% CI: 1.02-1.18; $P=0.0095)$. Late diagnosis was also associated with higher direct costs than early diagnosis.

Conclusion: Late COPD diagnosis is associated with higher exacerbation rate and increased comorbidities and costs compared with early diagnosis. The study highlights the need for accurate diagnosis of COPD in primary care in order to reduce exacerbations and the economic burden of COPD.

Keywords: chronic obstructive pulmonary disease, diagnosis, Sweden, exacerbations, mortality

\section{Background}

Chronic obstructive pulmonary disease (COPD) is often a progressive disease, characterized by persistent respiratory symptoms and airflow limitation, causing significant morbidity and mortality worldwide. ${ }^{1}$ COPD is associated with an economic, societal and personal burden that is evident at all stages of disease, ${ }^{2-6}$ and reflected by high rates of emergency department visits, hospitalizations and readmissions. $^{7-10}$ However, there is a direct relationship between COPD severity and the cost of care, which increases with disease progression. ${ }^{11,12}$

Despite an increased awareness and focus on COPD, there remains widespread under-recognition and under-diagnosis of the disease. ${ }^{13-15}$ A retrospective analysis 
of clinical data from 1990 to 2009 observed that opportunities for diagnosis were missed in $85 \%$ of 38,859 COPD patients in the 5 years preceding diagnosis. ${ }^{16}$ Although undiagnosed, these patients utilize health services to a similar degree as diagnosed patients, and contribute considerably to the health care burden of the disease. ${ }^{15}$ Many patients with chronic airflow limitation are conscious of symptoms for a number of years before seeking medical help ${ }^{17,18}$ and may misinterpret breathlessness, minor symptoms and low exercise tolerance as normal features of aging and as such do not seek medical help. ${ }^{19}$ However, initiating treatment early in the course of COPD is likely to slow disease progression and improve patientrelated outcomes. ${ }^{17}$

Therefore, it is widely recognized that it is beneficial for COPD patients to receive an early diagnosis. ${ }^{20,21}$ However, there are few studies directly comparing the effect of an early versus late COPD diagnosis on patientrelated outcomes and health care resource utilization. ${ }^{22}$ In an analysis of ARCTIC, a large real-world study, we investigated how the timing of COPD diagnosis is associated with clinical and economic outcomes in Swedish patients with COPD. There is no widely accepted method for identifying individuals who are at risk for development of COPD. Therefore, in this manuscript, we have identified factors that are associated with airways diseases (named indicators), which are likely predictors of a later COPD diagnosis.

\section{Methods}

\section{Study design}

This was a real-world, retrospective, observational cohort study of patients with COPD managed in primary care in Sweden. The study obtained ethical approval from the local Ethical Regional Board in Uppsala, Sweden, in December 2014 (number: 2014-397) and was conducted in accordance with the principles of the Declaration of Helsinki.

Electronic medical record (EMR) data were collected from COPD patients at 52 primary care centers across Sweden between 2000 and 2014, using an established software system (Pygargus Customized eXtraction Program [CXP]). Data collection included: age and gender, prescriptions (according to the World Health Organization [WHO] Anatomic Therapeutic Chemical [ATC] codes), diagnoses (according to the International Classification of Disease codes [ICD-9/ICD-10 codes]), spirometry measurements, laboratory tests, health care professional (HCP) visits and referrals. The centers covered urban and rural sites of varying size across Sweden. EMR data were linked using unique individual pseudonymized identification (ID) numbers to national registry data sources (see supplementary material).

\section{Study patients}

Patients eligible for inclusion were aged $\geq 40$ years who had received either a physician's diagnosis of COPD (ICD-10 code: J44) in primary care (EMR database), or a physician's diagnosis of asthma (ICD-10 code: J45/J46) in primary care that was later verified as COPD in the hospital setting (according to the National Patient Register).

Data from EMRs were examined for early recorded indicators that should attract the doctor's attention to a potential COPD diagnosis during the 5 years prior to date of diagnosis (index date). Such indicators were pneumonia (ICD10 code J10-J18), respiratory diseases other than pneumonia (ICD10 code J00-J99), prescription of oral steroids (ATC code H02AB), prescription of antibiotics for airway and lung infections (ATC codes J01AA, J01CA), prescriptions for respiratory disease targeted to relieve respiratory symptoms (ATC code R01-03 and R05-07) and lung function measurements (from spirometry database and lab module in EMR). Patients with $\leq 2$ registered indicators during the last 5 years prior to COPD diagnosis were classified as having an early diagnosis (ED) and patients with $\geq 3$ indicators (registered $>90$ days prior to the COPD diagnosis) during the 5 years prior to COPD diagnosis were classified as having a late COPD diagnosis (LD). A lag of time of 90 days prior to the index date was applied to ensure that no patients were incorrectly assigned to the LD group.

\section{Outcomes}

Outcomes were recorded in the 2-year period prior to, and following the index date. The annual rate of, and time to first exacerbation were recorded during the 2 years following index date. Exacerbations were defined as COPDrelated hospitalizations (J44 in primary position or J44.0/ $\mathrm{J} 44.1$ in secondary) and emergency visits (J44.0/J44.1 in outpatient hospital care), or collection of oral steroids (ATC $\mathrm{H} 02 \mathrm{AB}$ ), or antibiotics targeted at respiratory diseases (ARC J01AA/J01CA). Mortality risk was recorded as time to death and calculated from the index date. Comorbidities were defined through diagnosis codes in 
the primary or secondary care setting and by medications according to the national prescription register. Health care resource utilization (HCRU) 2 years prior to, and following the index date was assessed by: drug costs, outpatient/ inpatient COPD-related or unrelated hospital visits and primary care visits.

\section{Statistical analysis}

Patient demographics (corresponding to the 2-year period prior to index date) were reported descriptively and are stratified by diagnosis timing (early vs late). Exacerbation risk was analyzed using Cox regression with time to first exacerbation as the dependent variable and group (ED/LD) as a factor. Mortality risk was analyzed using Cox regression with time to death as dependent variable and group (ED vs LD) as a factor. Comorbidities were presented as the percentage of patients with each condition while frequencies of comorbidities were calculated relative to the index date. Overall comorbidity burden was assessed by the Charlson Comorbidity Index. Medication use was calculated as the percentage of patients with a prescription in the 2-year period prior to the index date. HCRU were calculated for each group and plotted graphically. Sensitivity analyses excluded patients with a comorbid asthma diagnosis at any time in the patient's disease history.

A sample size calculation conducted prior to the study indicated that 13,800 patients were required to detect a $4 \%$ difference between groups, with a power of $80 \%$ and a significance level of 5\%. Results are presented as ageadjusted mean values (95\% confidence interval [CI]) or hazard ratio (HR, $[95 \% \mathrm{CI}])$. Missing EMR data for essential risk factors were reviewed thoroughly in the data management process (eg, clinical measures, laboratory test results). Missing values were addressed by exclusion of non-essential variables, imputation based on average measures across the follow-up time frame or use of proxy algorithms. All analyses were performed using SAS version 9.3 or newer (SAS Institute Inc., Cary, NC) statistics software.

\section{Results}

\section{Patient demographics}

A total of 12,697 patients with a diagnosis of COPD were identified as eligible for inclusion, of which 3,870 (30.5\%) and $8,827(69.5 \%)$ were classified as $\mathrm{ED}$ or $\mathrm{LD}$, respectively (Figure 1). Mean ages were 68.7 and 69.5 years in ED and LD groups, respectively $(P=0.0005)$. Patients in the ED group were more likely to be male than female ( $53 \%$ vs $47 \%$ ); conversely, the proportion of females was higher compared with males (57\% vs $43 \%)$ in the LD group. Baseline characteristics are presented in Table 1.

\section{Exacerbations}

The annual exacerbation rate in the total population was 1.41 (95\% CI: 1.35-1.47) in the ED group and $2.67(95 \%$ CI: 2.60-2.74) in the LD group (HR 1.89, 95\% CI: $1.83-1.96 ; P<0.0001)$ measured 2 years after diagnosis. Time to first exacerbation also differed between the groups (HR 1.61, 95\% CI: 1.54-1.69; $P<0.0001$; Figure 2A, Table S1).

In patients with COPD only (ie, after exclusion of patients with a concomitant asthma diagnosis), the annual exacerbation rates were 1.37 (95\% CI: $1.31-1.44)$ and 2.53 (95\% CI: 2.43-2.63) exacerbations/year in those with ED and LD, respectively, and the time to first exacerbation was shorter in patients with LD than in patients with ED (HR 1.64, 95\% CI: 1.55-1.73; $P<0.0001$ : Figure 2C, Table S2). This higher exacerbation risk in the LD group also remained 2 years after index date in patients with only COPD (HR 1.59, 95\% CI: 1.51-1.67; $P<0.0001$ ).

Among patients with COPD and concomitant asthma, the annual exacerbation rate was 1.58 (95\% CI: $1.43-1.74)$ in ED and 2.85 (95\% CI: 2.74-2.96) in LD (HR 1.43, 95\% CI: 1.31-1.56 between groups; $P<0.0001$; Table 2). However, among patients with ED, the time to first exacerbation was longer in patients with COPD only compared with those with concomitant asthma (HR 0.89, 95\% CI: $0.81-0.97 ; P<0.05)$ whereas patients with only COPD and LD had an increased risk of exacerbations compared with ED patients with concomitant asthma (HR 1.41, 95\% CI: 1.30-1.54, $P<0.0001$; Table 2).

\section{Mortality}

The risk of mortality was similar between the ED and LD groups overall (HR 1.04, 95\% CI: 0.98-1.11; $P=0.1838$; Figure 2B, Table S1). However, after exclusion of patients with a concurrent asthma diagnosis, mortality was higher among patients in the LD group compared with those in the ED group (HR 1.10, 95\% CI: 1.02-1.18; $P=0.0095$ : Figure 2D, Table S2). Further, compared with ED patients with a comorbid asthma diagnosis, the risk of mortality was significantly higher among patients with COPD alone with both ED (HR 1.71, 95\% CI: 1.34-2.17; $P<0.0001$ ) and LD (HR 1.82, 95\% CI: 1.44-2.31; $P<0.0001$; Table 2). 


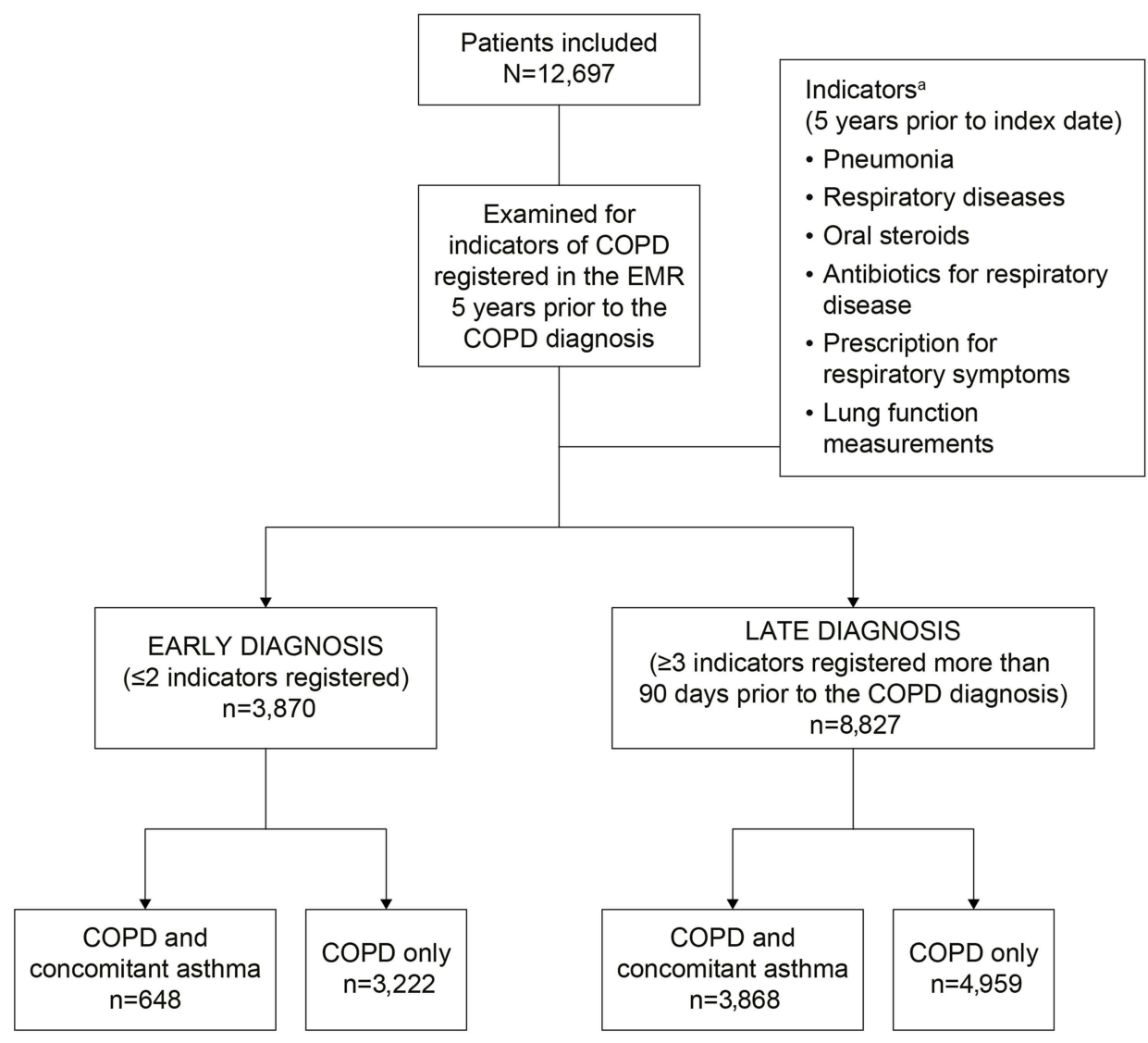

Figure I Study cohorts and criteria for early versus late COPD diagnosis. ${ }^{a}$ Indicator diagnosis codes; Pneumonia JI0-JI8; respiratory diseases J00-J99, excluding pneumonia; oral steroids H02AB; antibiotics for respiratory disease J0IAA, J0ICA; prescription for respiratory disease R0I-R03, R05-R07; lung function measurements from spirometry database and laboratory module in EMR. Disposition of patients by COPD and concomitant asthma versus COPD only is based on diagnosis at any time during the patient's disease history.

Abbreviation: EMR, electronic medical records.

\section{Comorbidities and use of medicines}

During the 2 years prior to diagnosis, patients in the LD group had a higher prevalence of comorbidities than those in the ED group. The Charlson Comorbidity Index (1.94 vs $1.59 ; P<0.0001)$ and the rates of cardiovascular disease, hypertension, type II diabetes, hyperlipidemia, depression, anxiety and osteoporosis were higher in the LD than in the ED group (Table 1).

Overall, 4,515 patients (35.6\%) had also received an asthma diagnosis at any time point in their disease history; $648(16.7 \%)$ patients in the ED group and 3,867 (43.8\%) patients in the LD group. Of these 4,515 patients, the asthma diagnosis was received 0-2 years prior to index date in $152(3.9 \%)$ patients in the ED group and 1,942 $(22.0 \%)$ in the LD group $(P<0.0001)$. Corresponding figures for the asthma diagnosis made more than 2 years prior to index date were $62(1.6 \%)$ patients in the ED group and $1,040(11.8 \%)$ patients in the LD group. This implies that $5.5 \%$ of the patients in the ED group and $33.8 \%$ of the patients in the LD group had asthma diagnosed before they received their COPD diagnosis. Asthma was diagnosed after index date in $434(11.3 \%)$ and $885(10.0 \%)$ patients in the ED and LD groups, respectively.

Compared with the ED group, significantly higher percentages of patients in the LD group used medications such as beta-blockers, calcium-channel blockers, reninangiotensin system modifiers, diuretics, lipid-modifying agents, diabetes medications, antidepressants and central pain-killers $(P<0.0001$ for all; Table 1). Furthermore, a significantly higher percentage of LD used oral steroids and antibiotics targeting respiratory infections compared with ED $(P<0.0001$ for both; Table 1$)$.

\section{Health care resource utilization}

During the 2 years prior to index date, patients in the LD group had a significantly higher number of primary care visits than patients in the ED group (Table 1). In the 2 years post-diagnosis, the cost per patient including drug 
Table I Patient demographics of the early versus late diagnosis populations

\begin{tabular}{|c|c|c|c|}
\hline Variable $^{a}$ & $\begin{array}{l}\text { Early diagnosis } \\
(n=3,870)\end{array}$ & $\begin{array}{l}\text { Late diagnosis } \\
(n=8,827)\end{array}$ & $p$-value \\
\hline Mean age (years) & 68.72 & 69.47 & 0.0005 \\
\hline $\begin{array}{l}\text { Gender, } \mathrm{n}(\%) \\
\text { Female } \\
\text { Male }\end{array}$ & $\begin{array}{ll}I, 82 I & (47.05) \\
2,049 & (52.95)\end{array}$ & $\begin{array}{l}5,074(57.48) \\
3,753(42.52)\end{array}$ & $<0.0001$ \\
\hline $\begin{array}{l}\text { Comorbidities } \\
\text { Number of Charlson Comorbidity groups }(95 \% \mathrm{Cl}) \\
\text { Charlson Comorbidity Index value }(95 \% \mathrm{Cl})\end{array}$ & $\begin{array}{l}1.48(1.46-1.52) \\
1.58(1.54-1.62)\end{array}$ & $\begin{array}{l}1.81(1.79-1.83) \\
1.94(1.91-1.97)\end{array}$ & $\begin{array}{l}<0.0001 \\
<0.0001\end{array}$ \\
\hline $\begin{array}{l}\text { Comorbidities by ICD-I0 code \% (95\% Cl) } \\
\text { Asthma, J45 } \\
\text { Rhinitis, J30/J3I } \\
\text { Cardiovascular diseases, 100-199 } \\
\text { Ischemic heart diseases, I20-125 } \\
\text { Hypertensive diseases, II0 } \\
\text { Cerebrovascular diseases, 160-169 } \\
\text { Diabetes type I, EI0 } \\
\text { Diabetes type II, EI I/EI3 } \\
\text { Hyperlipidemia, E78.5 } \\
\text { Depression, F32/F33 } \\
\text { Anxiety, F40/F4I } \\
\text { Mild cognitive impairment, F06.7 } \\
\text { Osteoporosis, M80/M8I } \\
\text { Fractures, S2 } \\
\text { Lung cancer, C34 }\end{array}$ & $\begin{array}{l}3.93(2.76-5.04) \\
0.54(0.14-0.92) \\
40.31(39.4-42.4) \\
10.78(9.99-12.0) \\
24.13(23.1-25.8) \\
4.88(4.31-5.70) \\
1.86(1.38-2.33) \\
6.77(5.94-7.75) \\
2.40(1.88-2.93) \\
4.81(3.95-5.50) \\
3.90(3.10-4.46) \\
0.34(0.13-0.58) \\
1.91(1.48-2.51) \\
6.82(6.04-7.70) \\
0.93(0.59-1.28)\end{array}$ & $\begin{array}{l}22.01(21.3-22.8) \\
2.02(1.76-2.28) \\
46.6(45.4-47.4) \\
12.41(11.6-13.0) \\
29.68(28.6-30.5) \\
5.29(4.78-5.70) \\
2.56(2.24-2.88) \\
10.13(9.50-10.7) \\
3.07(2.72-3.42) \\
7.28(6.81-7.84) \\
5.39(5.00-5.89) \\
0.60(0.44-0.74) \\
3.22(2.84-3.53) \\
7.76(7.19-8.29) \\
1.35(1.12-1.57)\end{array}$ & $\begin{array}{l}<0.0001 \\
<0.0001 \\
<0.0001 \\
0.009 \\
<0.0001 \\
0.3404 \\
0.0165 \\
<0.0001 \\
0.0381 \\
<0.0001 \\
<0.0001 \\
0.0564 \\
<0.0001 \\
0.0641 \\
0.0484\end{array}$ \\
\hline $\begin{array}{l}\text { Medication use by ATC code,\% }(95 \% \mathrm{Cl}) \\
\text { Treatment of cardiac disease, } \mathrm{COI} \\
\text { Antihypertensives, } \mathrm{C} 02 \\
\text { Beta-blocking agents, C07 } \\
\text { Calcium channel blockers, C08 } \\
\text { Agents acting on the renin-angiotensin system, C09 } \\
\text { Lipid modifying agents, CI0 } \\
\text { Diabetes medication, Al0 } \\
\text { Antidepressants, N06A } \\
\text { Central pain killers, N02BE/N02A/M0IAE } \\
\text { Bisphosphonates, M05BA } \\
\text { Proton pump inhibitors, A02 } \\
\text { Oral steroids, H02AB } \\
\text { Antibiotic for airways, J0IAA/J0ICA }\end{array}$ & $\begin{array}{l}13.13(12.3-14.27) \\
0.83(0.52-1.17) \\
25.99(24.9-27.8) \\
15.87(14.9-17.4) \\
24.86(23.8-26.7) \\
18.94(17.8-20.4) \\
7.62(6.68-8.68) \\
16.77(15.3-18.0) \\
28.32(26.9-30.0) \\
2.12(1.55-2.86) \\
16.5(15.1-17.9) \\
5.45(4.15-6.67) \\
14.19(12.6-15.5)\end{array}$ & $\begin{array}{l}18.55(17.6-19.2) \\
1.19(0.97-1.40) \\
33.70(32.6-34.5) \\
22.51(21.6-23.2) \\
36.04(34.9-36.8) \\
26.46(25.5-27.2) \\
13.15(12.5-13.8) \\
27.88(27.0-28.8) \\
50.38(49.3-51.3) \\
5.66(5.19-6.06) \\
34.6(33.7-35.5) \\
29.43(28.6-30.3) \\
46.55(45.7-47.6)\end{array}$ & $\begin{array}{l}<0.0001 \\
0.0687 \\
<0.0001 \\
<0.0001 \\
<0.0001 \\
<0.0001 \\
<0.0001 \\
<0.0001 \\
<0.0001 \\
<0.0001 \\
<0.0001 \\
<0.0001 \\
<0.0001\end{array}$ \\
\hline $\begin{array}{l}\text { Health care utilization } \\
\text { Hospital visits per year for any reason, } \mathrm{n} \\
\text { Outpatient }{ }^{\mathrm{b}} \text { visits per year, } \mathrm{n} \\
\text { Primary care contacts per year, } \mathrm{n} \\
\text { Patients with primary care contacts, } \% \\
\text { Patients with outpatient hospital visits, } \% \\
\text { Patients with overnight stays,\% }\end{array}$ & $\begin{array}{l}0.41(0.37-0.45) \\
1.36(1.21-1.47) \\
7.76(7.22-8.41) \\
78.4(77.1-79.7) \\
57.2(55.7-58.6) \\
33.0(31.7-34.7)\end{array}$ & $\begin{array}{l}0.56(0.54-0.59) \\
2.24(2.16-2.33) \\
13.3(12.9-13.4) \\
77.9(77.0-78.7) \\
72.1(71.1-73.1) \\
39.7(38.6-40.6)\end{array}$ & $\begin{array}{l}<0.0001 \\
<0.0001 \\
<0.0001 \\
0.4739 \\
<0.0001 \\
<0.0001\end{array}$ \\
\hline
\end{tabular}

Notes: ${ }^{2}$ Variables quoted for 2 years prior to COPD diagnosis (index) date unless otherwise stated; all variables except age itself are calculated with age as a covariate. bOutpatient visit: a visit where the patient does not stay overnight at the hospital.

Abbreviations: ATC, anatomical therapeutic chemical; Cl, confidence interval; COPD, chronic obstructive pulmonary disease; ICD-I0, international classification of disease code. 

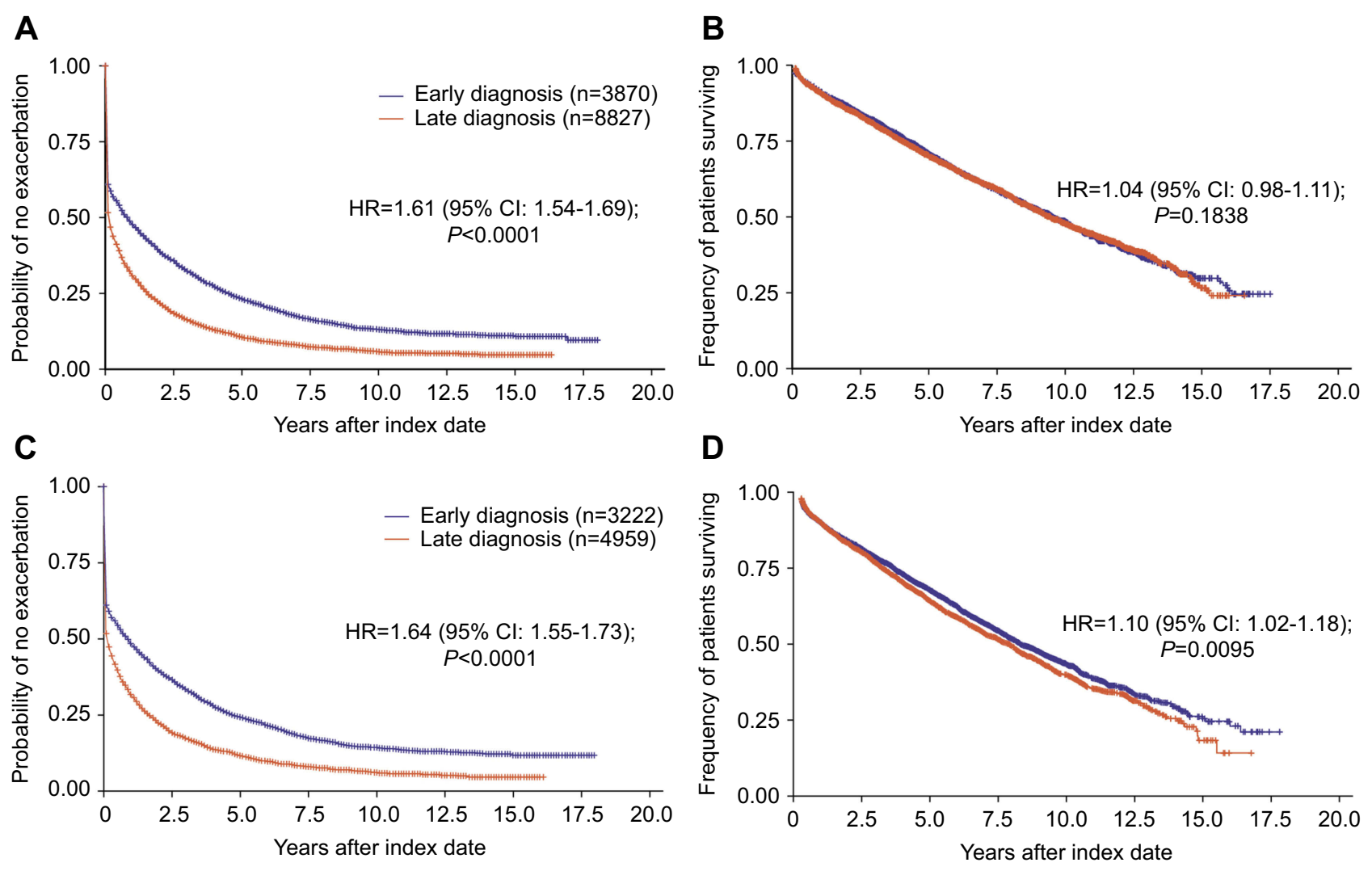

Figure 2 Risk of exacerbations and mortality by stage of diagnosis (early vs late). Risk of exacerbations (A) and mortality (B) in the total population and risk of exacerbations (C) and mortality (D) in patients excluding those with concomitant asthma, in the year following diagnosis.

Table 2 Influence of asthma on time to first exacerbation and mortality, early versus late COPD diagnosis

\begin{tabular}{|l|l|l|l|l|}
\hline Outcome & $\begin{array}{l}\text { All patients } \\
\text { late diagnosis } \\
(\mathrm{n}=\mathbf{8 , 8 2 7})\end{array}$ & $\begin{array}{l}\text { Late diagnosis concomitant asthma } \\
(\mathrm{n}=3,868)\end{array}$ & \multicolumn{2}{|l|}{ COPD-only } \\
\cline { 2 - 5 } & $\begin{array}{l}\text { Early diagnosis } \\
(\mathrm{n}=3,222)\end{array}$ & $\begin{array}{l}\text { Late diagnosis } \\
(\mathrm{n}=4,959)\end{array}$ \\
\hline Column heading versus early diagnosis with concomitant asthma $(\mathrm{n}=648)$ & $0.89(0.81-0.97)^{\mathrm{b}}$ & $1.41(1.30-1.54)^{\mathrm{a}}$ \\
\hline Exacerbation & $1.42(1.31-1.55)^{\mathrm{a}}$ & $1.43(1.31-1.56)^{\mathrm{a}}$ & $1.71(1.34-2.17)^{\mathrm{a}}$ & $1.82(1.44-2.31)^{\mathrm{a}}$ \\
\hline Mortality & $1.41(1.11-1.80)^{\mathrm{b}}$ & $1.42(1.12-1.81)^{\mathrm{b}}$ &
\end{tabular}

Notes: ${ }^{a} P<0.000$ I; ${ }^{b} P<0.05$. Data are for the 2 years following COPD diagnosis, presented as HR ( $95 \%$ confidence interval), patients with early versus late diagnosis. Concomitant asthma includes patients with a comorbid asthma diagnosis.

Abbreviations: COPD, chronic obstructive pulmonary disease; HR, hazard ratio.

costs and primary and secondary care contacts was $€ 22,526$ and $€ 19,481$ in the LD and ED groups, respectively. In a time trend analysis, costs were consistently higher for LD versus ED, with differences achieving significance at four out of eight time points measured (Figure 3, Table S3). These differences were driven largely by costs associated with respiratory drugs and COPDrelated hospital nights (Table 3, Figure 4, Table S4).
There were no significant differences in the annual number of primary care contacts and hospital visits between ED with concomitant asthma and ED with COPD only during 2 years preceding diagnosis (Table 4). However, LD patients with concomitant asthma had a significantly higher number of primary care visits than LD patients with COPD only and a significantly higher percentage of LD patients with concomitant asthma had more primary care 


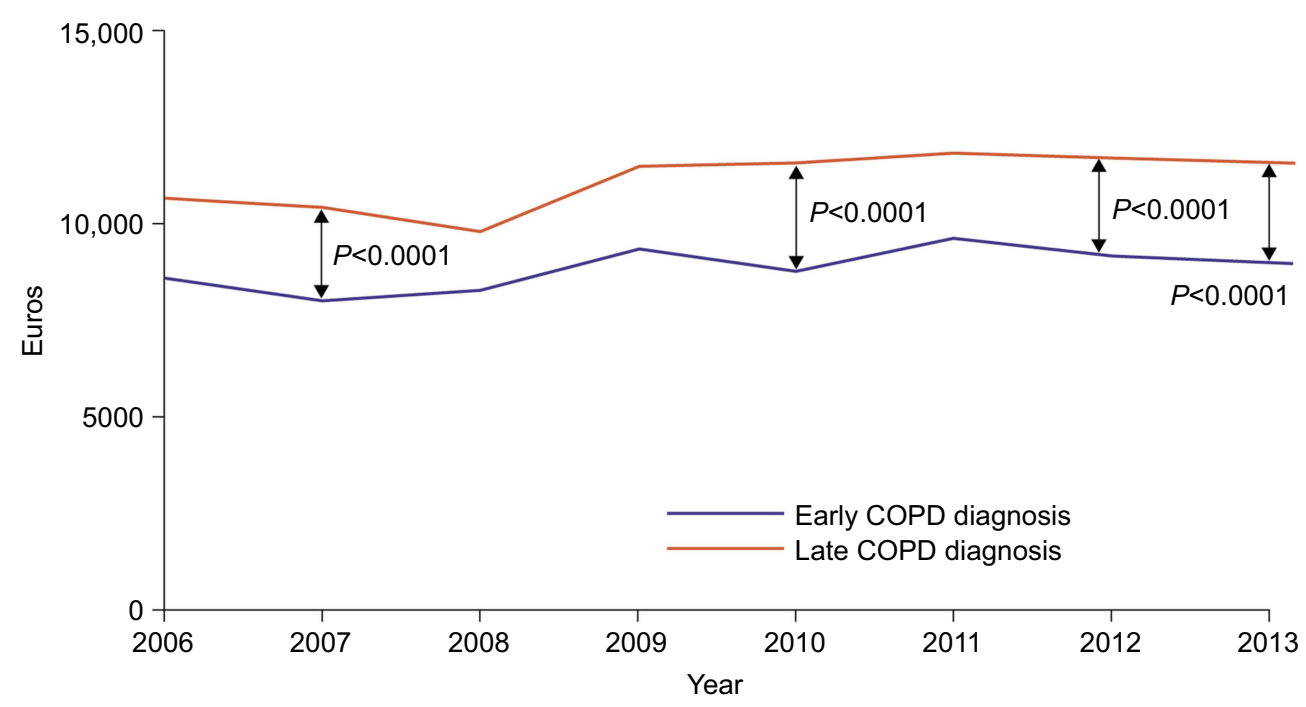

Figure 3 Time trend in direct costs (Euro/year) according to the stage of diagnosis (early vs late). In the year 2007, 2010, 2012 and 2013, total yearly costs were significantly higher for late diagnosis versus early diagnosis.

Table 3 Direct health care costs and utilization in patients with early versus late COPD diagnosis

\begin{tabular}{|c|c|c|c|}
\hline Variable $^{a}$ & $\begin{array}{l}\text { Early diagnosis } \\
(\mathrm{N}=3,870)\end{array}$ & $\begin{array}{l}\text { Late diagnosis } \\
(\mathrm{N}=\mathbf{8 , 8 2 7})\end{array}$ & p-value \\
\hline \multicolumn{4}{|l|}{ Cost per patient, Euro $(95 \% \mathrm{Cl})$} \\
\hline Total for respiratory drugs (R03) & $386.5(353.7-419.4)$ & $900.1(878.3-921.8)$ & $<0.0001$ \\
\hline Public cost & $314.0(283.9-344.1)$ & 782.1 (762.I-802) & $<0.000$ I \\
\hline Individual patient cost & $72.6(68.4-76.8)$ & $118.0(115.3-120.8)$ & $<0.0001$ \\
\hline Total drug cost per patient & $460.8(396.6-524.9)$ & $716.6(674.2-759.1)$ & $<0.0001$ \\
\hline Public cost & $373.2(310.4-436.0)$ & $595.3(553.7-636.8)$ & $<0.0001$ \\
\hline Individual patient cost & $87.6(80.0-95.3)$ & $121.4(116.4-126.5)$ & $<0.0001$ \\
\hline \multicolumn{4}{|l|}{ Utilization per patient per year, $\mathrm{n}(95 \% \mathrm{Cl})$} \\
\hline Nights at hospital (COPD-related; J45) & $0.16(0.03-0.38)$ & $1.17(1.02-1.30)$ & $<0.0001$ \\
\hline Nights at hospital (non-COPD) & $11.65(11.04-12.55)$ & $11.58(11.01-12.02)$ & 0.5487 \\
\hline Hospitalization events (COPD-related; J44) & $0.03(0.01-0.05)$ & $0.17(0.16-0.18)$ & $<0.0001$ \\
\hline Hospitalization events (non-COPD) & $1.68(1.59-1.78)$ & $1.79(1.72-1.85)$ & 0.0933 \\
\hline Out hospital visits ${ }^{\mathrm{b}}$ (COPD-related; J45) & $0.04(0.02-0.06)$ & $0.15(0.14-0.16)$ & $<0.0001$ \\
\hline Out hospital visits $^{\mathrm{b}}$ (non-COPD) & $3.76(3.44-4.02)$ & $4.74(4.56-4.94)$ & $<0.0001$ \\
\hline Primary care contact with physician & $13.06(|2.4|-13.73)$ & $15.77(15.33-16.21)$ & $<0.000$ I \\
\hline Primary care contact with persons other than physician & $9.20(8.42-9.98)$ & $12.48(11.96-13.00)$ & $<0.0001$ \\
\hline Number of referrals & $0.99(0.91-1.06)$ & $1.22(1.17-1.28)$ & $<0.0001$ \\
\hline
\end{tabular}

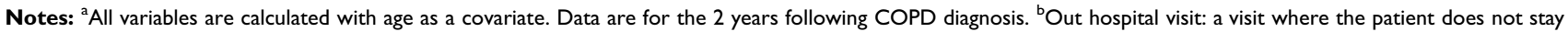
overnight at the hospital. R03 indicates the ATC prescription code for respiratory disease. Exchange rates in 20I3, 8 Swedish Krona= I Euro.

contacts and overnight stays than LD patients with COPD only (Table 4).

\section{Discussion}

The present study shows that receiving an early COPD diagnosis is associated with significant benefits compared with receiving a diagnosis later in the course of the disease. Compared with a LD, early COPD diagnosis was associated with fewer exacerbations, fewer comorbidities and lower health care utilization in the 2 years following index date. Based on the present data, receiving a COPD diagnosis soon after having visited a doctor due to airway symptoms is 


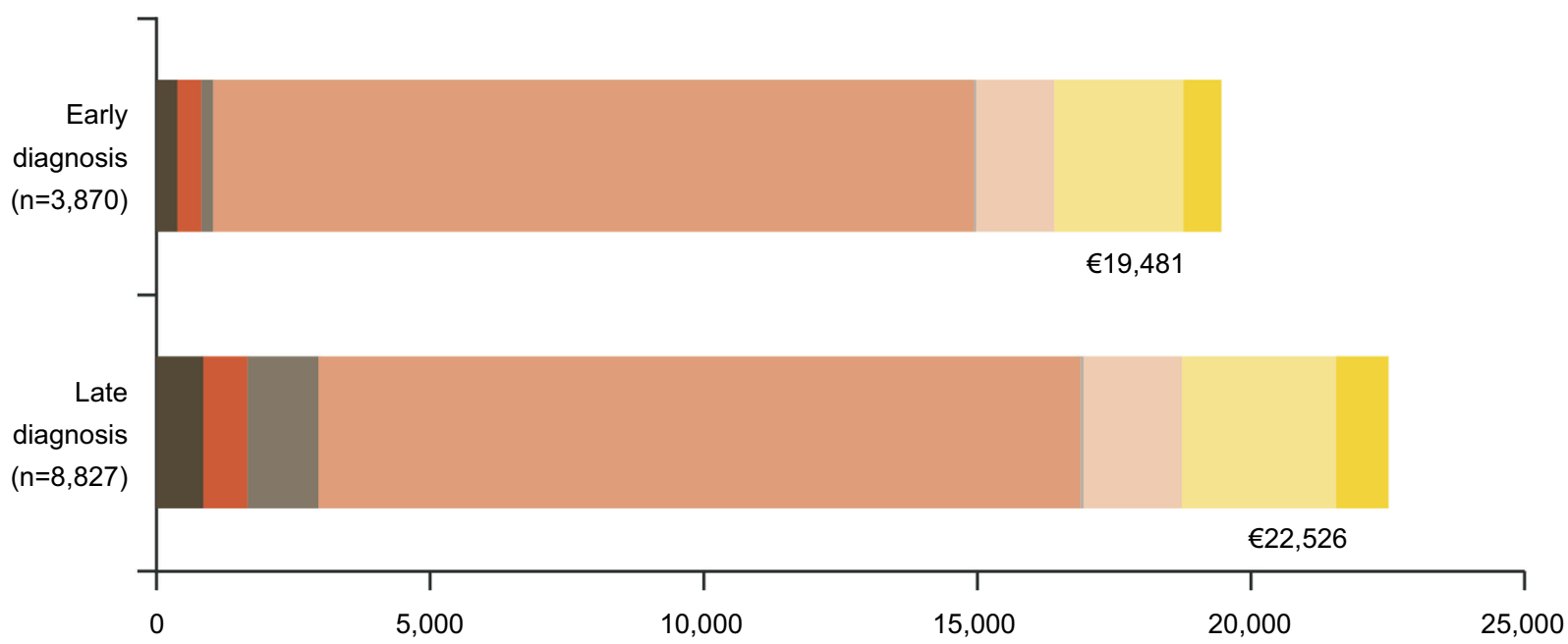

Direct costs (during 2 years after COPD diagnosis, Euros)

Drugs, respiratory

Hospital nights, not COPD

Primary care contacts, physician
Drugs, other

Hospital visits, COPD

Primary care contacts, not physician

Figure 4 Direct costs during the 2 years after COPD diagnosis by early vs late diagnosis. Costs were calculated from Swedish Krona based on exchange rates in 2013. Abbreviations: hospital nights, number of nights where the patient has been at the hospital; hospital visit, outpatient care where the patient did not stay overnight at the hospital.

Table 4 Health care resource utilization stratified by a concomitant diagnosis of asthma, early versus late diagnosis

\begin{tabular}{|c|c|c|c|c|c|c|}
\hline \multirow[t]{2}{*}{ Variable $^{a}$} & \multicolumn{3}{|l|}{ Early diagnosis } & \multicolumn{3}{|l|}{ Late diagnosis } \\
\hline & $\begin{array}{l}\text { Concomitant } \\
\text { asthma } \\
(\mathbf{N}=648)\end{array}$ & $\begin{array}{l}\text { COPD } \\
\text { only } \\
(N=3,222)\end{array}$ & $p$-value ${ }^{b}$ & $\begin{array}{l}\text { Concomitant } \\
\text { asthma } \\
(\mathbf{N}=\mathbf{3 , 8 6 8 )}\end{array}$ & $\begin{array}{l}\text { COPD } \\
\text { only } \\
(N=4,959)\end{array}$ & $p$-value ${ }^{b}$ \\
\hline Mean age, years & 66.27 & 69.21 & $<0.0001$ & 68.51 & 70.22 & $<0.001$ \\
\hline $\begin{array}{l}\text { Number of hospital visits per year for } \\
\text { any reason }\end{array}$ & 0.35 & 0.42 & 0.0912 & 0.60 & 0.53 & 0.0225 \\
\hline $\begin{array}{l}\text { Number of out hospital visits } \\
\text { per yearc }\end{array}$ & 1.11 & 1.41 & 0.0168 & 2.20 & 2.27 & 0.4461 \\
\hline $\begin{array}{l}\text { Number of primary care contacts } \\
\text { per year }\end{array}$ & 7.44 & 7.82 & 0.4790 & 15.97 & 11.16 & $<0.0001$ \\
\hline Patients with overnight stays, $\%$ & 28.70 & 33.80 & 0.0118 & 41.11 & 38.66 & 0.0196 \\
\hline Patients with primary care contact,\% & 79.48 & 78.21 & 0.4758 & 82.01 & 74.61 & $<0.000$ I \\
\hline
\end{tabular}

Notes: ${ }^{a}$ All variables except age itself are calculated with age as a covariate. Data are for the 2 years prior to COPD diagnosis. ${ }^{b} p$-values indicate comparisons between patients with a concomitant diagnosis of asthma and those with COPD-only. ${ }^{c}$ Out hospital visit: a visit where the patient does not stay overnight at the hospital.

associated with better clinical and economic outcomes than receiving the COPD diagnosis later during the course of the disease despite earlier presence of indicators of airway disease.

It is unclear why the majority of patients in the present study received an LD of COPD. Patients may delay seeking treatment for symptoms in some instances; however, we found that indicators suggestive of chronic airway disease had been present and registered by a medical professional $>90$ days prior to the receipt of a COPD diagnosis in all LD patients. It is likely that a delay in diagnosis in the present study was due to initial misdiagnosis by HCPs. Additionally, time-pressured doctor-patient consultations, a low general 
awareness of COPD and insufficient local routines for COPD care may have contributed. ${ }^{23}$ It is also possible that patient comorbidities require more attention from a treating physician, thereby moving the focus away from COPD symptoms, resulting in a delayed COPD diagnosis.

Patients with chronic airflow obstruction frequently have features of both asthma and COPD, a condition sometimes called asthma-COPD overlap, and often have both an asthma and a COPD diagnosis. Patients with asthma-COPD overlap, particularly those with late-onset asthma, seem to have more frequent exacerbations and poor prognosis compared with patients who have only asthma or COPD. ${ }^{24,25}$ In the present study, asthma was much more common in the LD group than in the ED group. While similar proportions of patients received an asthma diagnosis after index date in the ED and LD groups, six times more patients in the LD group than in the ED group received an asthma diagnosis prior to index date.

This may have influenced the results in two ways. Firstly, in patients who already had an asthma diagnosis, the responsible doctor may not have considered the possibility of COPD, a diagnosis that was therefore missed. Secondly, as some patients had asthma before index date, this may have increased the number of registered "COPD indicators" (prescription due to respiratory symptom, lung function assessment and to some extent oral steroid treatment), measures which may have been taken due to asthma and therefore not regarded as direct predictors of COPD. On the other hand, all patients included in our analysis eventually received a COPD diagnosis, most of them within 2 years after the asthma diagnosis. This suggests that the indicators should also have been considered as predictive indicators of COPD. These findings indicate that there may be a need for increased awareness of the coexistence between asthma and COPD and that COPD should be considered in adult patients who are under treatment for asthma at primary health care centers.

It could be argued that pre-existing asthma may increase the risk of overestimating the occurrence of LD. However, this did not influence our main results as the main outcomes were similar after also excluding patients with asthma from the analyses. Further, data support the beneficial effects of early treatment in COPD. ${ }^{17}$ It is therefore imperative that primary care physicians are aware of the clinical indicators associated with COPD, in order to facilitate the early identification and treatment of patients with the disease. Even patients with mild or moderate COPD can suffer from substantial limitations and physical impairment which worsen with disease progression, ${ }^{22}$ and the rate of forced expiratory volume in 1 second decline is faster in patients with mild and moderate COPD than in those with very severe disease. $^{26,27}$ Furthermore, the early treatment of acute exacerbations in COPD is associated with a faster recovery and the prevention of future exacerbations versus a delay in intervention, while failure to report acute exacerbations is associated with impaired health-related quality of life and increased hospitalization. ${ }^{28}$

Patients with ED with concomitant asthma were at higher risk and experienced a higher annual rate of exacerbations than patients with COPD only, suggesting that a concomitant asthma diagnosis increases the risk of exacerbations even in the mild stages of COPD. A strong association between asthma and chronic bronchitis in "middle-aged" (early to mid-forties) adults has previously been demonstrated. ${ }^{29}$ Furthermore, as chronic bronchitis is associated with increased number and severity of exacerbations, ${ }^{30}$ it is tempting to speculate that this could have contributed to the increased risk of exacerbation observed in patients with concomitant asthma compared with COPD only.

Although there was no overall difference in mortality between ED and LD, mortality was higher in LD than in ED in patients with COPD only, ie, after exclusion of patients with a concomitant asthma diagnosis. The presence of asthma appears to "dilute" the negative effect of late COPD diagnosis on mortality, a finding that is supported by the significant differences in mortality found when results were stratified according to asthma diagnosis. In both ED and LD, patients with concomitant asthma had a significantly lower rate of mortality than patients without concomitant asthma. Patients with features of both asthma and COPD (asthmaCOPD overlap) are known to experience more frequent exacerbations, a poorer quality of life, a more rapid decline in lung function and a higher rate of mortality than those with either asthma or COPD alone. ${ }^{24,31}$ It is likely that ED patients diagnosed with concomitant asthma had a mild or moderate COPD component to disease; however, some patients may have suffered from severe asthma. A less-severe COPD component in patients with concomitant asthma compared with COPD only patients could provide one explanation for the observed differences in mortality.

We found that LD patients suffered more frequently from comorbidities such as cardiovascular disease, depression, osteoporosis and diabetes than ED patients. It is well known that there is an association between COPD and a number of comorbidities, which may have an impact 
on the course of the disease, and that COPD can adversely affect the outcome of other disorders. For example, there is a greater rate of morbidity and mortality in hospitalized patients undergoing cardiac procedures when COPD is present compared with when it is absent. ${ }^{32}$ The presence of other diseases could have contributed to an LD of COPD as physicians may have given greater priority to the treatment of other morbidities, overlooking or misdiagnosing the respiratory symptoms indicative of COPD. For example, symptoms of breathlessness in patients with heart failure or lung cancer could have been overlooked, while fatigue and reduced physical activity could have been disregarded in patients with depression. ${ }^{33}$

We also observed that health care utilization and total drug cost were significantly higher in LD patients compared with ED. This finding is likely to relate to the higher prevalence of comorbidities in LD patients, in line with previous studies which have shown annual costs to be higher in COPD patients with comorbidities than in those without. ${ }^{34}$ In LD patients, the presence of concomitant asthma led to a significantly higher rate of primary care contacts and percentage of patients with overnight stays, consistent with evidence that patients with both asthma and COPD features experience poorer outcomes than those with either asthma or COPD alone, ${ }^{24,31}$ and will utilize a greater proportion of health care resources.

This study has a number of strengths. The large sample size of COPD patients from a primary care setting and the robust outcomes of this study provide data that are highly representative of the general population. ${ }^{35-37}$ Furthermore, the cohort study design allows for the investigation of a temporal relationship between outcome variables which otherwise would not have been possible to investigate in a cross-sectional study. ${ }^{38}$ Although there is no accepted method by which to define an ED and a LD, we believe that identifying indicators of respiratory symptoms, or disease, in patients who later received a COPD diagnosis was a credible approach to the identification of undiagnosed COPD. Furthermore, introducing a 90-day lag time prior to the date of diagnosis allowed an acceptable period of time for physician investigation, and ensured that no patients were incorrectly assigned to the LD group.

A potential limitation to this study is the retrospective study design, which introduces the potential for bias and confounding. As only Swedish patients were enrolled, this may limit the generalizability of these results to a more diverse group of patients and to different health care systems. ${ }^{39}$ However, we believe our findings have important clinical implications. If an earlier COPD diagnosis is associated with improved outcomes and reductions in the overall costs associated with the disease, primary care physicians have a responsibility to detect the early signs of COPD, perform relevant tests in a timely manner, make an accurate diagnosis and treat patients appropriately.

\section{Clinical implications and conclusion}

In conclusion, early COPD diagnosis is associated with a lower risk of exacerbations, fewer comorbidities and lower costs. Additionally, there may be mortality benefits of accurately differentiating asthma from COPD. This study indicates that an earlier diagnosis may lead to improved clinical outcomes while concomitantly reducing the economic burden of COPD. The study also indicates that a pre-existing asthma diagnosis may increase the risk of missing a COPD diagnosis.

\section{Abbreviation list}

ATC, anatomic therapeutic chemical; CI, confidence interval; COPD, chronic obstructive pulmonary disease; CXP, customized eXtraction program; ED, early diagnosis; EMR, electronic medical record; HCP, health care professional; HCRU, health care resource utilization; HR, hazard ration; ICD, international classification of disease; ID, identification; LD, late diagnosis; PC, primary care; WHO, World Health Organization.

\section{Availability of data and materials}

The datasets used and/or analyzed during the current study are available from the corresponding author on reasonable request.

\section{Ethics approval and consent to participate}

The study obtained ethical approval from the local Ethical Regional Board in Uppsala, Sweden, in December 2014 (number: 2014-397). An amendment was approved in October 2017. This study was conducted in accordance with the principles of the Declaration of Helsinki

\section{Acknowledgments}

The authors were assisted in the preparation of the manuscript by Emily Fisher and Mary Sayers, professional medical writers at CircleScience, an Ashfield Company, part of UDG Health care plc. The statistical analyses and medical writing support were funded by Novartis Pharma AG (Basel, 
Switzerland). KK was an employee of Novartis Pharma AG at the time the study was conducted.

\section{Author contributions}

$\mathrm{KjL}, \mathrm{J}-\mathrm{BG}, \mathrm{CJ}, \mathrm{GJ}, \mathrm{KK}, \mathrm{LJ}, \mathrm{KL}, \mathrm{PO}$ and BS made substantial contributions to the conception and design of the study as well as the analysis and interpretation of the results. FSG made substantial contributions to the analysis and interpretation of the results of the study. MU and LJ analyzed the data for the study. All authors made substantial contributions to the drafting of the manuscript, revising it critically for important intellectual content, approved the final version to be published and agree to be accountable for all aspects of the work, ensuring that questions related to the accuracy or integrity of any part of the work are appropriately investigated and resolved.

\section{Disclosure}

$\mathrm{KjL}$ has, during the last 5 years, on one or more occasion served in an advisory board and/or served as speaker and/or participated in education arranged by AstraZeneca, Boehringer Ingelheim, GlaxoSmithKline, Orion, Takeda, Novartis, Chiesi and TEVA. CJ has received honoraria for educational activities and lectures from AstraZeneca, Boehringer Ingelheim, Chiesi, Novartis and TEVA, and has served on advisory boards arranged by AstraZeneca, TEVA and Boehringer Ingelheim. BS has received honoraria for educational activities and lectures from AstraZeneca, Boehringer Ingelheim, GlaxoSmithKline, Novartis, MEDA and TEVA, and has served on advisory boards arranged by AstraZeneca, Novartis, GlaxoSmithKline and Boehringer Ingelheim. KL has participated in the steering committee by Novartis for this study and received honoraria for educational activities and lectures from AstraZeneca, GlaxoSmithKline, Novartis, MEDA and TEVA and has served on advisory boards arranged by MEDA and Novartis. FSG and JBG are employees of Novartis Pharma AG. PO is an employee of Novartis AB. MU and LJ are employees of IQVIA, who received remuneration in relation to statistical analysis. GJ has participated in the steering committee by Novartis for this study and served on advisory boards arranged by AstraZeneca, Novo Nordisk and Takeda. BS reports personal fees from Novartis, during the conduct of the study; received personal fees from AstraZeneca, Novartis, Meda, Teva, Boehringer Ingelheim, and GlaxosSmithKline, outside the submitted work; KL reports personal fees from
Novartis, during the conduct of the study; received personal fees from AstraZeneca, Novartis, Chiesi, TEVA, GlaxoSmithKline, and Boerhringer Ingelheim, outside the submitted work; KK was an employee of Novartis, the sponsor of the study, at the time of conduct of this analysis and until 31.10.2018. He is now affiliated with Respiratory Medicine Department, University of Ioannina, Ioannina, Greece. KK has received honoraria for educational activities and lectures from AstraZeneca, Boehringer Ingelheim, Chiesi, ELPEN, GlaxoSmithKline, MSD, Novartis, Takeda, and UCB, and has served on advisory boards arranged by AstraZeneca, Chiesi, ELPEN, Novartis and Takeda prior to 2015. The authors report no other conflicts of interest in this work.

\section{References}

1. Global Initiative for Chronic Obstructive Lung Disease (GOLD) [webpage from Internet]. Global strategy for the diagnosis, management, and prevention of chronic obstructive pulmonary disease; 2018. Available from: http:/goldcopd.org/gold-reports/. Accessed November 28, 2018.

2. Vos T, Flaxman AD, Naghavi M, et al. Years lived with disability (YLDs) for 1160 sequelae of 289 diseases and injuries 1990-2010: a systematic analysis for the Global Burden of Disease Study 2010. Lancet. 2012;380(9859):2163-2196. doi:10.1016/S0140-6736(12) 61729-2

3. Lozano R, Naghavi M, Foreman K, et al. Global and regional mortality from 235 causes of death for 20 age groups in 1990 and 2010: a systematic analysis for the Global Burden of Disease Study 2010. Lancet. 2012;380(9859):2095-2128. doi:10.1016/S0140-6736(12) 61728-0

4. Lopez AD, Shibuya K, Rao C, et al. Chronic obstructive pulmonary disease: current burden and future projections. Eur Respir J. 2006;27 (2):397-412. doi:10.1183/09031936.06.00025805

5. Mathers CD, Loncar D. Projections of global mortality and burden of disease from 2002 to 2030. PLoS Med. 2006;3(11):e442. doi:10.1371/journal.pmed.0030442

6. Murray CJ, Lopez AD. Alternative projections of mortality and disability by cause 1990-2020: Global Burden of Disease Study. Lancet. 1997;349(9064):1498-1504. doi:10.1016/S0140-6736(96) 07492-2

7. May SM, Li JT. Burden of chronic obstructive pulmonary disease: healthcare costs and beyond. Allergy Asthma Proc. 2015;36(1):4-10. doi:10.2500/aap.2015.36.3815

8. Benfield T, Lange P, Vestbo J. COPD stage and risk of hospitalization for infectious disease. Chest. 2008;134(1):46-53. doi:10.1378/ chest.07-2933

9. Troosters T, Sciurba F, Battaglia S, et al. Physical inactivity in patients with COPD, a controlled multi-center pilot-study. Respir Med. 2010;104(7):1005-1011. doi:10.1016/j.rmed.2010.01.012

10. de Marco R, Accordini S, Cerveri I, et al. An international survey of chronic obstructive pulmonary disease in young adults according to GOLD stages. Thorax. 2004;59(2):120-125. doi:10.1136/ thorax.2003.011163

11. Jansson SA, Backman H, Stenling A, Lindberg A, Ronmark E, Lundback B. Health economic costs of COPD in Sweden by disease severity - has it changed during a ten years period? Respir Med. 2013;107(12):1931-1938. doi:10.1016/j.rmed.2013.07.012 
12. Stallberg B, Janson C, Johansson G, et al. Management, morbidity and mortality of COPD during an 11-year period: an observational retrospective epidemiological register study in Sweden (PATHOS). Prim Care Respir J. 2014;23(1):38-45.

13. Lindberg A, Bjerg A, Rönmark E, Larsson LG, Lundbäck B. Prevalence and underdiagnosis of COPD by disease severity and the attributable fraction of smoking report from the obstructive lung disease in Northern Sweden studies. Respir Med. 2006;100(2):264272. doi:10.1016/j.rmed.2006.03.043

14. Sundblad BM, Larsson K, Nathell L. Low awareness of COPD among physicians. Clin Respir J. 2008;2(1):11-16. doi:10.1111/ j.1752-699X.2007.00020.x

15. Labonte LE, Tan WC, Li PZ, et al. Undiagnosed chronic obstructive pulmonary disease contributes to the burden of health care use. Data from the CanCOLD Study. Am J Respir Crit Care Med. 2016;194 (3):285-298. doi:10.1164/rccm.201509-1795OC

16. Jones RC, Price D, Ryan D, et al. Opportunities to diagnose chronic obstructive pulmonary disease in routine care in the UK: a retrospective study of a clinical cohort. Lancet Respir Med. 2014;2(4):267-276. doi:10.1016/S2213-2600(14)70008-6

17. Welte T, Vogelmeier C, Papi A. COPD: early diagnosis and treatment to slow disease progression. Int J Clin Pract. 2015;69(3):336-349. doi:10.1111/ijcp.2015.69.issue-3

18. Global Initiative for Chronic Obstructive Lung Disease (GOLD) [webpage from Internet]. Global strategy for the diagnosis, management, and prevention of chronic obstructive pulmonary disease; 2017. Available from: http://goldcopd.org/gold-2017-global-strategydiagnosis-management-prevention-copd/. Accessed January 3, 2017.

19. Decramer M, Miravitlles M, Price D, et al. New horizons in early stage COPD-improving knowledge, detection and treatment. Respir Med. 2011;105(11):1576-1587. doi:10.1016/j.rmed.2011.03.020

20. Csikesz NG, Gartman EJ. New developments in the assessment of COPD: early diagnosis is key. Int J Chron Obstruct Pulmon Dis. 2014;9:277-286. doi:10.2147/COPD.S60179

21. Leff RD. COPD: clinical significance of early diagnosis. J Manag Care Pharm. 2005;11(6 Suppl A):S8-11;quiz S20-12.

22. Maltais F, Dennis N, Chan CK. Rationale for earlier treatment in COPD: a systematic review of published literature in mild-tomoderate COPD. Copd. 2013;10(1):79-103. doi:10.3109/ 15412555.2012.719048

23. Sandelowsky H, Hylander I, Krakau I, Modin S, Stallberg B, Nager A. Time pressured deprioritization of COPD in primary care: a qualitative study. Scand J Prim Health Care. 2016;34(1):55-65. doi: 10.3109/02813432.2015.1132892

24. Gibson PG, McDonald VM. Asthma-COPD overlap 2015: now we are six. Thorax. 2015;70(7):683-691. doi:10.1136/thoraxjnl-2014-206740

25. Lange P, Colak Y, Ingebrigtsen TS, Vestbo J, Marott JL. Long-term prognosis of asthma, chronic obstructive pulmonary disease, and asthma-chronic obstructive pulmonary disease overlap in the Copenhagen City Heart study: a prospective population-based analysis. Lancet Respir Med. 2016;4(6):454-462. doi:10.1016/ S2213-2600(16)00098-9
26. Drummond MB, Hansel NN, Connett JE, Scanlon PD, Tashkin DP, Wise RA. Spirometric predictors of lung function decline and mortality in early chronic obstructive pulmonary disease. Am J Respir Crit Care Med. 2012;185(12):1301-1306. doi:10.1164/rccm.201202$0223 \mathrm{OC}$

27. Tantucci C, Modina D. Lung function decline in COPD. Int J Chron Obstruct Pulmon Dis. 2012;7:95-99.

28. Tm W, Donaldson GC, Hurst JR, Seemungal TA, Wedzicha JA. Early therapy improves outcomes of exacerbations of chronic obstructive pulmonary disease. Am J Respir Crit Care Med. 2004;169(12):12981303.

29. Dharmage SC, Perret JL, Burgess JA, et al. Current asthma contributes as much as smoking to chronic bronchitis in middle age: a prospective population-based study. Int J Chron Obstruct Pulmon Dis. 2016;11:1911-1920.

30. Han MK, Kazerooni EA, Lynch DA, et al. Chronic obstructive pulmonary disease exacerbations in the COPDGene study: associated radiologic phenotypes. Radiology. 2011;261(1):274-282. doi:10. 1148/radiol.11110173

31. Kostikas K, Clemens A, Patalano F. The asthma-COPD overlap syndrome: do we really need another syndrome in the already complex matrix of airway disease? Int J Chron Obstruct Pulmon Dis. 2016;11:1297-1306.

32. Mascarenhas J, Lourenco P, Lopes R, Azevedo A, Bettencourt P. Chronic obstructive pulmonary disease in heart failure. Prevalence, therapeutic and prognostic implications. Am Heart J. 2008;155 (3):521-525. doi:10.1016/j.ahj.2007.10.040

33. Agusti A, Calverley PM, Celli B, et al. Characterisation of COPD heterogeneity in the ECLIPSE cohort. Respir Res. 2010;11:122. doi:10.1186/1465-9921-11-62

34. Wouters EF. Economic analysis of the Confronting COPD survey: an overview of results. Respir Med. 2003;97(Suppl.C):S3-S14. doi:10.1016/S0954-6111(03)80020-3

35. Lisspers $\mathrm{K}$, Johansson $\mathrm{G}$, Jansson $\mathrm{C}$, et al. Improvement in COPD management by access to asthma/COPD clinics in primary care: data from the observational PATHOS study. Respir Med. 2014;108(9):1345-1354. doi:10.1016/j.rmed.2014.06. 002

36. Holgate S, Bisgaard H, Bjermer L, et al. The Brussels Declaration: the need for change in asthma management. Eur Respir J. 2008;32 (6):1433-1442. doi:10.1183/09031936.00053108

37. Pinnock H, Thomas M, Tsiligianni I, et al. The International Primary Care Respiratory Group (IPCRG) research needs statement 2010. Prim Care Respir J. 2010;19 (Suppl 1): S1-20.

38. Song JW, Chung KC. Observational studies: cohort and case-control studies. Plast Reconstr Surg. 2010;126(6):2234-2242. doi:10.1097/ PRS.0b013e3181dbc2f0

39. Atsou K, Chouaid C, Hejblum G. Variability of the chronic obstructive pulmonary disease key epidemiological data in Europe: systematic review. BMC Med. 2011;9:7. doi:10.1186/ 1741-7015-9-7 


\section{Supplementary materials}

\section{Methods}

\section{National registry data sources}

Electronic medical record (EMR) data were linked using unique individual pseudonymized identification (ID) numbers to national registry data sources.

These included:

1. Longitudinal Integration Database for Health Insurance and Labor Market Studies (LISA), which contains sociodemographic data, including: educational level, marital status and family situation, occupational status, retirement, economic compensation and social benefits

2. National Patient Register, which contains data relating to diagnosis (International Classification of Disease
[ICD]-10 code and associated position): surgery, gender, age, region, hospital visits, specialty visits, hospital admissions and discharges, and medical procedures and surgeries performed in inpatient and outpatient specialist settings

3. National Prescription Register, which contains the full details of all dispensed medications (Anatomic Therapeutic Chemical [ATC] codes) from both primary and secondary care including: brand name, prescription date, dose, strength, pack size, specialty of the prescriber and costs associated with the drug prescription

4. Cause of Death Register, which holds information relating to sex, date of death and underlying cause of death.

\section{Results}

Table SI Risk of exacerbations and mortality according to the stage of diagnosis (early versus late): total population

\begin{tabular}{|l|l|l|l|l|}
\hline \multirow{2}{*}{ Years since index date } & \multicolumn{2}{|l|}{$\begin{array}{l}\text { Risk of exacerbation, percentage of patients } \\
\text { without event } \mathbf{9 5 \%} \mathbf{~ C I})\end{array}$} & \multicolumn{2}{l|}{$\begin{array}{l}\text { Risk of mortality, percentage of patients } \\
\text { without event (95\% CI) }\end{array}$} \\
\cline { 2 - 5 } & Early diagnosis & Late diagnosis & Early diagnosis & Late diagnosis \\
\hline 2.5 & $35.0(33.5-36.5)$ & $18.1(17.3-18.9)$ & $83.7(82.5-84.9)$ & $82.6(81.8-83.5)$ \\
5.0 & $22.7(21.3-24.1)$ & $10.7(10.0-11.4)$ & $71.5(69.9-73.0)$ & $69.6(68.5-70.7)$ \\
7.5 & $16.2(14.9-17.5)$ & $7.4(6.7-8.1)$ & $59.4(57.6-61.2)$ & $58.9(57.6-60.3)$ \\
10.0 & $12.8(11.6-14.1)$ & $5.8(5.2-6.5)$ & $48.8(46.8-50.8)$ & $48.1(46.4-49.7)$ \\
12.5 & $11.4(10.2-12.7)$ & $5.1(4.4-5.8)$ & $38.7(36.5-41.0)$ & $39.1(37.1-41.0)$ \\
15.0 & $10.5(9.2-11.9)$ & $4.7(4.0-5.5)$ & $30.8(28.0-33.6)$ & $30.8(28.2-33.5)$ \\
17.5 & $10.0(8.5-11.6)$ & $4.7(4.0-5.5)$ & $24.4(20.2-28.9)$ & $24.8(19.7-30.1)$ \\
\hline
\end{tabular}

Abbreviation: $\mathrm{Cl}$, confidence interval.

Table S2 Risk of exacerbations and mortality according to the stage of diagnosis (early versus late): patients excluding those with concomitant asthma (COPD-only population)

\begin{tabular}{|c|c|c|c|c|}
\hline \multirow[t]{2}{*}{ Years since index date } & \multicolumn{2}{|c|}{$\begin{array}{l}\text { Risk of exacerbation, percentage of patients } \\
\text { without event }(95 \% \mathrm{Cl})\end{array}$} & \multicolumn{2}{|c|}{$\begin{array}{l}\text { Risk of mortality, percentage of patients } \\
\text { without event }(95 \% \mathrm{CI})\end{array}$} \\
\hline & Early diagnosis & Late diagnosis & Early diagnosis & Late diagnosis \\
\hline 2.5 & $35.9(34.3-37.6)$ & $19.0(17.9-20.2)$ & $81.6(80.1-82.9)$ & $80.2(79.0-81.4)$ \\
\hline 5.0 & $24.0(22.4-25.5)$ & $12.0(11.0-13.1)$ & $68.6(66.9-70.3)$ & $65.3(63.7-66.9)$ \\
\hline 7.5 & $17.4(15.9-18.9)$ & $8.3(7.3-9.4)$ & $56.1(54.0-58.1)$ & $53.9(51.9-55.8)$ \\
\hline 10.0 & $14.2(12.8-15.6)$ & $6.3(5.3-7.4)$ & $45.6(43.3-47.8)$ & $42.9(40.5-45.3)$ \\
\hline 12.5 & $12.8(\mid 1.4-14.3)$ & $5.4(4.4-6.6)$ & $36.3(33.7-38.9)$ & $34.5(31.6-37.5)$ \\
\hline 15.0 & $11.7(10.1-13.3)$ & $4.8(3.6-6.2)$ & $29.7(26.4-33.1)$ & $24.3(20.0-28.9)$ \\
\hline 17.5 & $11.7(10.1-13.3)$ & $4.8(3.6-6.2)$ & $24.7(19.6-30.1)$ & $20.6(13.5-28.8)$ \\
\hline
\end{tabular}

Abbreviations: $\mathrm{Cl}$, confidence interval; COPD, chronic obstructive pulmonary disease. 
Table S3 Time trend in direct overall costs (Euro/year) according to the stage of diagnosis (early versus late)

\begin{tabular}{|l|l|l|l|}
\hline Year & $\begin{array}{l}\text { Total, } \\
\epsilon / \text { year }\end{array}$ & $\begin{array}{l}\text { Early diagnosis, } \\
\epsilon / \text { year }\end{array}$ & $\begin{array}{l}\text { Late diagnosis, } \\
\epsilon / \text { year }\end{array}$ \\
\hline 2006 & $9,776.4$ & $8,474.2$ & 10,835 \\
2007 & $9,450.4$ & $7,890.3$ & 10,656 \\
2008 & $9,202.5$ & $8,515.4$ & $9,692.2$ \\
2009 & 10,715 & $9,346.9$ & 11,611 \\
2010 & 10,626 & $8,825.2$ & 11,673 \\
2011 & 11,126 & $9,687.3$ & 11,871 \\
2012 & 10,947 & $9,138.5$ & 11,816 \\
2013 & 10,859 & $8,994.9$ & 11,677 \\
\hline
\end{tabular}

Table S4 Health care resource utilization direct costs over 2 years after index date according to stage of diagnosis (late vs early)

\begin{tabular}{|l|l|l|}
\hline & Early diagnosis, $€$ & Late diagnosis, $€$ \\
\hline Drugs, respiratory (R03) & 386.5 & 900.1 \\
Drugs, other & 460.8 & 716.6 \\
Hospital nights, COPD (45) & 191.6 & $1,397.48$ \\
Hospital nights, not COPD & $13,944.89$ & $13,865.85$ \\
Hospital visits, COPD (45) & 15.0 & 56.25 \\
Hospital visits, not COPD & $1,410.0$ & $1,777.5$ \\
Primary care contacts, physician & $2,386.72$ & $2,881.97$ \\
Primary care contacts, not physician & 685.4 & 929.76 \\
\hline
\end{tabular}

Notes: Costs were based on unit costs for hospital nights (9,580 SEK), hospital visits (3,000 SEK), primary care visits, physician ( 1,462 SEK) and primary care visits, nurse (596 SEK) and converted to Euros based on exchange rates in 2013 (8/1).

Abbreviations: COPD, chronic obstructive pulmonary disease; SEK, Swedish Krona; Hospital nights, number of nights where the patient has been at the hospital; hospital visit, outpatient care where the patient did not stay overnight at the hospital.

\section{Publish your work in this journal}

The International Journal of COPD is an international, peer-reviewed journal of therapeutics and pharmacology focusing on concise rapid reporting of clinical studies and reviews in COPD. Special focus is given to the pathophysiological processes underlying the disease, intervention programs, patient focused education, and self management protocols. This journal is indexed on PubMed Central, MedLine and CAS. The manuscript management system is completely online and includes a very quick and fair peer-review system, which is all easy to use. Visit http://www.dovepress.com/testimonials.php to read real quotes from published authors. 